\title{
Many-variable variational Monte Carlo study of superconductivity in two-band Hubbard models with an incipient band
}

\author{
Daichi Kato* and Kazuhiko Kuroki ${ }^{\dagger}$ \\ Department of Physics, Osaka University, 1-1 Machikaneyama, Toyonaka, Osaka 560-0043, Japan
}

(Received 27 December 2019; revised manuscript received 9 April 2020; accepted 14 April 2020; published 11 May 2020)

\begin{abstract}
We study superconductivity in two-band models where one of the bands does or does not intersect the Fermi level depending on the parameter values. Applying a many-variable variational Monte Carlo method for a Hubbard model on two-leg ladder and bilayer square lattices, we show that superconductivity can be enhanced in a parameter regime where the edge of one of the bands is near the Fermi energy, that is, when the band is incipient. The resemblance of the present results to those obtained by a weak-coupling method in a recent study suggests that, even in the large- $U$ regime, the suppression of the near-zero-energy spin fluctuations and the development of finite-energy spin fluctuations are the key factors for the enhancement of superconductivity by an incipient band.
\end{abstract}

DOI: 10.1103/PhysRevResearch.2.023156

\section{INTRODUCTION}

A purely electronic mechanism of superconductivity is expected to exhibit extremely high $T_{\mathrm{c}}$ due to the large energy scale of the pairing glue originating from quantum fluctuations, such as spin, charge, and orbital fluctuations. In particular, spin-fluctuation-mediated pairing is one of the leading candidate mechanisms at work in unconventional high-temperature superconductors, namely, cuprates and ironbased superconductors.

In particular, in the early days of the study of iron-based superconductors, it was considered that the Fermi surface nesting between electron and hole Fermi surfaces, combined with Hubbard $U$, induces spin fluctuations, which in turn act as a pairing interaction around a certain wave vector $\boldsymbol{Q}$ if the gap sign changes across $\boldsymbol{Q}$. This kind of superconducting gap is referred to as the $s^{ \pm}$pairing [1-6]. However, the spin-fluctuation theory has been challenged by the discovery of (heavily) electron-doped iron-based superconductors with a relatively high $T_{\mathrm{c}}$ where holelike bands sink below the Fermi level leaving only electronlike Fermi surfaces [7-19]. Naively, removing the hole pocket is expected to destroy the spin-fluctuation-mediated pairing interaction and suppress $T_{\mathrm{c}}$ rapidly.

After these observations, "incipient bands," which sit close to but do not intersect the Fermi level, have received much attention. Various authors have suggested that the spin-fluctuation scattering of pairs between an electron Fermi surface and an incipient hole band can induce $s^{ \pm}$

\footnotetext{
*d.kato@ presto.phys.sci.osaka-u.ac.jp

${ }^{\dagger}$ kuroki@phys.sci.osaka-u.ac.jp

Published by the American Physical Society under the terms of the Creative Commons Attribution 4.0 International license. Further distribution of this work must maintain attribution to the author(s) and the published article's title, journal citation, and DOI.
}

pairing $[1,15,17,20-25]$. In this context, the bilayer Hubbard model, which has been extensively studied in the past [26-35], has recently attracted renewed focus. Having hole and electron Fermi surfaces, it can be regarded as a single-orbital analog of the iron-based superconductors. In fact, it has been found in previous studies that $s^{ \pm}$pairing is favored over $d_{x^{2}-y^{2}}$ pairing by increasing the relative strength of the interlayer nearest hopping to the intralayer nearest hopping [36]. Furthermore, as one of the bands becomes shallow or incipient, the spectral weight of spin fluctuation is transferred to higher energies, which can lead to an $s^{ \pm}$pairing state in which a gap appears on the hole band with the opposite sign to the gap on the electron Fermi surfaces [37-39].

Regarding the incipient band situation, it was proposed in Ref. [40] by one of the present authors and his co-workers that strongly enhanced superconductivity can take place in a system with coexisting wide and narrow bands when the narrow band sits in the vicinity of the Fermi level. There, the Hubbard model on a two-leg ladder was studied within the fluctuation exchange (FLEX) approximation. In the twoleg ladder model, which is a two-band model with bonding and antibonding bands, one of the bands becomes wide and the other becomes narrow when diagonal hoppings are introduced. In today's terminology, the narrow band in this case is incipient. Quite recently, partially motivated by studies on various lattice models with coexisting wide and narrow (or flat) bands [41-46], one of the present authors and his co-workers studied the bilayer model with diagonal interlayer hoppings [47], where one of the bands becomes wide and the other narrow, as in the two-leg ladder. There again, it has been shown using the FLEX approximation that $s^{ \pm}$-wave superconductivity is strongly enhanced when one of the bands is incipient. The role of the finite- and low-energy spin fluctuations on superconductivity, along with the commonalities and differences with the two-leg ladder, has been discussed [47].

The above-mentioned studies on the two-leg ladder and bilayer lattices with diagonal hoppings adopted the FLEX 


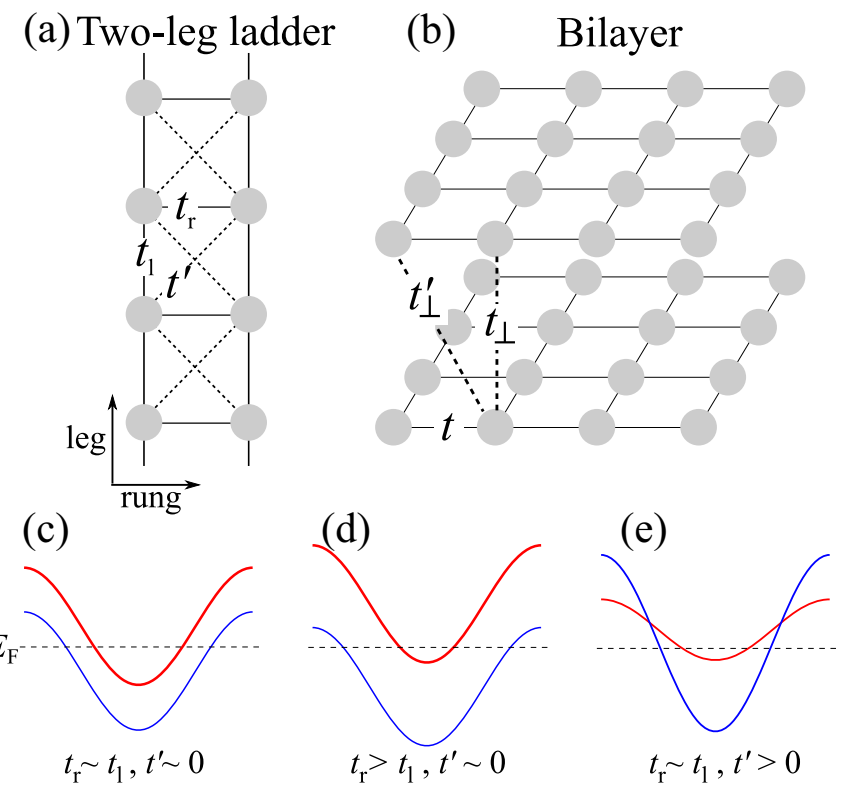

FIG. 1. (a) The two-leg ladder lattice and (b) the bilayer lattice. Lower panels: typical band structures of the two-leg ladder lattice. (c) $t_{\mathrm{r}} \sim t_{1}, t^{\prime} \sim 0$, (d) $t_{\mathrm{r}}>t_{1}, t^{\prime} \sim 0$, (e) $t_{\mathrm{r}} \sim t_{1}, t^{\prime}>0$.

approximation $[40,44,47]$, but because FLEX is basically a weak-coupling method, it is not clear whether the method can be applied to regimes where the electron-electron interaction is large. In the present study, we study Hubbard models on the two-leg ladder and bilayer square lattices, using a manyvariable variational Monte Carlo (mVMC) method [48,49], which can be considered reliable in the strong-coupling regime [50]. By comparing the results for the two-leg ladder (one dimensional) and the bilayer lattice (two dimensional), and with and without the diagonal hoppings, we discuss how the density of states (DOS) affects superconductivity and antiferromagnetism when one of the bands is close to being incipient.

\section{MODELS, METHOD, AND DEFINITIONS OF PHYSICAL QUANTITIES}

We study Hubbard models on the two-leg ladder and bilayer square lattices (Fig. 1). The Hamiltonian for the two-leg Hubbard ladder is

$$
\begin{aligned}
H= & -t_{1} \sum_{\langle i j\rangle m \sigma}\left(c_{i m \sigma}^{\dagger} c_{j m \sigma}+\text { H.c. }\right)-t_{\mathrm{r}} \sum_{i \sigma}\left(c_{i 0 \sigma}^{\dagger} c_{i 1 \sigma}+\text { H.c. }\right) \\
& -t^{\prime} \sum_{\langle i j\rangle \sigma}\left(c_{i 0 \sigma}^{\dagger} c_{j 1 \sigma}+\text { H.c. }\right)+U \sum_{i m} n_{i m \uparrow} n_{i m \downarrow} .
\end{aligned}
$$

Here $c_{i m \sigma}^{\dagger}\left(c_{i m \sigma}\right)$ creates (annihilates) a fermion with spin $\sigma(=\uparrow, \downarrow)$ on the $i$ th site on the $m$ th chain ( $m=0$ or 1$)$ and $n_{i m \sigma}=c_{i m \sigma}^{\dagger} c_{i m \sigma}$. The nearest-neighbor hoppings in the leg and rung directions are $t_{1}$ and $t_{\mathrm{r}}$, respectively, and the nextnearest-neighbor diagonal hopping is $t^{\prime}$. Since $t_{\mathrm{r}}$ connects two chains, we call this the interchain hopping. The band structure for this model is

$$
\varepsilon(\boldsymbol{k})=-2\left(t_{1}+t^{\prime} \cos k_{y}\right) \cos k_{x}-t_{\mathrm{r}} \cos k_{y},
$$

where the case of $k_{y}=0(\pi)$ corresponds to the bonding (antibonding) band. For $t^{\prime}>0$, the bonding band is wide and the antibonding band is narrow.

The Hamiltonian for the square lattice bilayer Hubbard model is

$$
\begin{aligned}
H= & -t \sum_{\langle i j\rangle m \sigma}\left(c_{i m \sigma}^{\dagger} c_{j m \sigma}+\text { H.c. }\right)-t_{\perp} \sum_{i \sigma}\left(c_{i 0 \sigma}^{\dagger} c_{i 1 \sigma}+\text { H.c. }\right) \\
& -t_{\perp}^{\prime} \sum_{\langle i j\rangle \sigma}\left(c_{i 0 \sigma}^{\dagger} c_{j 1 \sigma}+\text { H.c. }\right)+U \sum_{i m} n_{i m \uparrow} n_{i m \downarrow} .
\end{aligned}
$$

Here $c_{i m \sigma}^{\dagger}\left(c_{i m \sigma}\right)$ creates (annihilates) a fermion with spin $\sigma(=\uparrow, \downarrow)$ on the $i$ th site on the $m$ th layer $(m=0$ or 1$)$. The intralayer hopping is $t$, the interlayer hopping is $t_{\perp}$, and the next-nearest-neighbor interlayer hopping is $t_{\perp}^{\prime}$. The band structure for this model is

$$
\varepsilon(\boldsymbol{k})=-2\left(t+t_{\perp}^{\prime} \cos k_{z}\right)\left(\cos k_{x}+\cos k_{y}\right)-t_{\perp} \cos k_{z},
$$

where the case of $k_{z}=0(\pi)$ corresponds to the bonding (antibonding) band. For $t_{\perp}^{\prime}>0$, the bonding band is wide and the antibonding band is narrow.

We take $N_{\mathrm{s}}=60 \times 2(12 \times 12 \times 2)$ sites for the two-leg ladder (bilayer) Hubbard model with the antiperiodic-periodic boundary condition in the $x(y)$ direction. The band filling is defined as $n=N_{\mathrm{e}} / N_{\mathrm{s}}$, where $N_{\mathrm{e}}=\sum_{\text {mi }} n_{\text {im } \sigma}$. Hereinafter, the site index $(i, m)$ is simply written as $i$. We employ antiperiodic boundary conditions in order to study physical properties near half filling, avoiding the open-shell problem. In finite-size systems, highly degenerate states (open shell) are realized at specific band fillings, which can give rise to unstable optimization in the quantum Monte Carlo method. Open shell tends to occur near half filling, where superconductivity can be strongly enhanced, under periodic boundary conditions. On the other hand, if antiperiodic boundary conditions are used, the open-shell problem near half filling is remarkably reduced.

To study the ground state of these Hubbard models, we employ a mVMC method [48,49]. The variational Monte Carlo (VMC) method is free from the negative-sign problem unlike the auxiliary field quantum Monte Carlo method, so it can be applicable to relatively large systems even with strong electron correlation and geometrical frustration at reasonable computational cost. Furthermore, in the conventional VMC method, several dozen variational parameters are used, but in the present $\mathrm{mVMC}$ method, thousands of variational parameters can be employed, which describe various quantum fluctuations of order parameters and strong correlation effects accurately. Our variational wave function is defined as

$$
|\phi\rangle=\mathcal{P}_{\mathrm{G}} \mathcal{P}_{\mathrm{J}}\left|\phi_{\text {pair }}\right\rangle,
$$

where $\mathcal{P}_{\mathrm{G}}$ and $\mathcal{P}_{\mathrm{J}}$ are the Gutzwiller and Jastrow correlation factors, respectively. The Gutzwiller factor punishes the double occupation of electrons defined as

$$
\mathcal{P}_{\mathrm{G}}=\exp \left(-\frac{1}{2} \sum_{i} g_{i} n_{i \uparrow} n_{i \downarrow}\right) .
$$

The Jastrow factor is defined as

$$
\mathcal{P}_{\mathrm{J}}=\exp \left(-\frac{1}{2} \sum_{i j} v_{i j} n_{i} n_{j}\right),
$$


where $n_{i}=\sum_{\sigma} n_{i \sigma}$. The long-range part of this factor drives the distinction between the metal and insulator. $\left|\phi_{\text {pair }}\right\rangle$ is the one-body part defined as

$$
\left|\phi_{\text {pair }}\right\rangle=\left[\sum_{i, j=1}^{N_{\mathrm{s}}} f_{i j} c_{i \uparrow}^{\dagger} c_{j \downarrow}^{\dagger}\right]^{N_{\mathrm{e}} / 2}|0\rangle,
$$

where $f_{i j}$ is assumed to have $2 \times 2(2 \times 2 \times 2)$ sublattice structure or equivalently $2 \times 2 \times N_{\mathrm{s}}\left(2 \times 2 \times 2 \times N_{\mathrm{s}}\right)$ independent variational parameters for the one-body part in twoleg ladder (bilayer) systems.

To study a possible superconducting state, we consider the following BCS wave function,

$$
\left|\phi_{\mathrm{BCS}}\right\rangle=\left(\sum_{\boldsymbol{k} \in \mathrm{BZ}} \varphi(\boldsymbol{k}) c_{\boldsymbol{k} \uparrow}^{\dagger} c_{-\boldsymbol{k} \downarrow}^{\dagger}\right)^{N_{\mathrm{e}} / 2}|0\rangle,
$$

with

$$
\varphi(\boldsymbol{k})=\frac{\Delta(\boldsymbol{k})}{\xi(\boldsymbol{k})+\sqrt{\xi(\boldsymbol{k})^{2}+\Delta(\boldsymbol{k})^{2}}},
$$

where $\xi(\boldsymbol{k})=\varepsilon(\boldsymbol{k})-\mu, \mu$ is the chemical potential, and $\Delta(\boldsymbol{k})$ is the superconducting gap. The BCS wave function is rewritten in the real-space representation as follows:

$$
f_{i j}=\frac{1}{N_{\mathrm{s}}} \sum_{\boldsymbol{k}} \varphi(\boldsymbol{k}) \exp \left[i \boldsymbol{k} \cdot\left(\boldsymbol{r}_{i}-\boldsymbol{r}_{j}\right)\right] .
$$

In this study, we employ the BCS partial $d$-wave $\left(s^{ \pm}\right.$wave) superconducting state as the initial states for the ladder (bilayer) system, namely, $\Delta(\boldsymbol{k})=\Delta_{0} \cos k_{y}\left(\Delta_{0} \cos k_{z}\right)$. The variational parameters are simultaneously optimized to minimize the variational energy by using the stochastic reconfiguration method [51].

To investigate the ground-state properties of these Hubbard models, we calculate the momentum distribution function, spin-structure factor, and equal-time superconducting correlations. The momentum distribution function is defined as

$$
n_{\sigma}(\boldsymbol{q})=\frac{1}{N_{\mathrm{s}}} \sum_{i, j}\left\langle c_{i \sigma}^{\dagger} c_{j \sigma}\right\rangle \exp \left[i \boldsymbol{q} \cdot\left(\boldsymbol{r}_{i}-\boldsymbol{r}_{j}\right)\right]
$$

and the spin-structure factor is defined as

$$
S(\boldsymbol{q})=\frac{1}{3 N_{\mathrm{s}}} \sum_{i, j}\left\langle\boldsymbol{S}_{i} \cdot \boldsymbol{S}_{j}\right\rangle \exp \left[i \boldsymbol{q} \cdot\left(\boldsymbol{r}_{i}-\boldsymbol{r}_{j}\right)\right] .
$$

Regardless of the strength of the electron correlation, the momentum dependence of $S(\boldsymbol{q})$ is as follows: When both bands intersect the Fermi level, the spin-structure factor $S(\boldsymbol{q})$ is sharply peaked at a specific momentum $\boldsymbol{q}_{\max }$. Near half filling, where the present calculations are performed, $\boldsymbol{q}_{\max }$ is $(\pi, \pi)$ for the two-leg ladder and $(\pi, \pi, \pi)$ for the bilayer model. On the other hand, the peak structure of $S(\boldsymbol{q})$ becomes less and less pronounced as one of the bands moves away from the Fermi level. Furthermore, the equal-time superconducting correlations are defined as

$$
P_{\alpha}(\boldsymbol{r})=\frac{1}{2 N_{\mathrm{s}}} \sum_{\boldsymbol{r}_{i}}\left\langle\Delta_{\alpha}^{\dagger}\left(\boldsymbol{r}_{i}\right) \Delta_{\alpha}\left(\boldsymbol{r}_{i}+\boldsymbol{r}\right)+\Delta_{\alpha}\left(\boldsymbol{r}_{i}\right) \Delta_{\alpha}^{\dagger}\left(\boldsymbol{r}_{i}+\boldsymbol{r}\right)\right\rangle .
$$

Superconducting order parameters $\Delta_{\alpha}\left(\boldsymbol{r}_{i}\right)$ are defined as

$$
\Delta_{\alpha}\left(\boldsymbol{r}_{i}\right)=\frac{1}{\sqrt{2}} \sum_{\boldsymbol{r}} f_{\alpha}(\boldsymbol{r})\left(c_{\boldsymbol{r}_{i} \uparrow} c_{\boldsymbol{r}_{i}+\boldsymbol{r} \downarrow}-c_{\boldsymbol{r}_{i} \downarrow} c_{\boldsymbol{r}_{i}+\boldsymbol{r} \uparrow}\right) .
$$

Here $f_{\alpha}(\boldsymbol{r})$ is the form factor that describes the symmetry of the superconductivity. For the partial $d$-wave superconductivity in two-leg ladder systems, we define

$$
f_{d}\left(r_{x}, r_{y}\right)=\delta_{r_{x}, 0} \delta_{r_{y}, 1},
$$

where $\delta_{i j}$ denotes Kronecker's delta. For the $s^{ \pm}$-wave superconductivity in bilayer systems, we define

$$
f_{s^{ \pm}}\left(r_{x}, r_{y}, r_{z}\right)=\delta_{r_{x}, 0} \delta_{r_{y}, 0} \delta_{r_{z}, 1} .
$$

To reduce stochastic errors, we calculate the long-range average of the superconducting correlation, which is defined as

$$
\bar{P}_{\alpha}=\frac{1}{M} \sum_{2<|\boldsymbol{r}|<r_{\max }} P_{\alpha}(\boldsymbol{r}),
$$

where $r_{\max }$ is $30(6 \sqrt{2})$ for the present two-leg ladder (bilayer) models. $M$ is the number of vectors satisfying $2<r<r_{\max }$. Here, we eliminate the short-range part of the superconducting correlation since it does not reflect the off-diagonal ordering nature of superconductivity in order to reduce the effect of the boundary condition.

\section{RESULTS}

\section{A. Two-leg Hubbard ladder}

We begin with the two-leg ladder. Figure 2(a) shows the interchain hopping dependence of several physical properties for $t^{\prime} / t_{1}=0$ and $U / t_{1}=4$ : the peak value of the spin-structure factor $S\left(\boldsymbol{q}_{\max }\right)$, which is the square of the antiferromagnetic ordered moment, and the average value of superconducting correlation $\bar{P}_{d}$ at long distance with the partial $d$ symmetry, corresponding to the square of the superconducting order parameter. We also plot the momentum distribution function at the antibonding band minimum $n(0, \pi)$ [the bonding band maximum $n(\pi, 0)$ ], which monitors whether or not the antibonding (bonding) band intersects the Fermi level. For $1.2 \leqslant$ $t_{\mathrm{r}} / t_{1} \leqslant 1.6, n(0, \pi)$ decreases rapidly, which indicates that the antibonding band loses its Fermi points. On the other hand, $n(\pi, 0)$ increases in this $t_{\mathrm{r}} / t_{1}$ regime, but only moderately, which indicates that, although the Fermi level is approaching the top of the bonding band (actually it is close to the band top because the band filling $n=0.97$ is very close to half filling), it still intersects the band. Also within this $t_{\mathrm{r}} / t_{1}$ regime, $S\left(\boldsymbol{q}_{\max }\right)$ rapidly decreases. Therefore, the incipient band regime is estimated to be in the range $1.2 \leqslant t_{\mathrm{r}} / t_{1} \leqslant 1.6$. In the incipient band regime, $\bar{P}_{d}$ is maximized. Furthermore, the partial $d$ wave superconducting phase exhibits a dome structure around $t_{\mathrm{r}} / t_{1} \sim 1.5$, which is reminiscent of a previous FLEX study on the two-leg ladder Hubbard model without $t^{\prime}$ [52]. For $t^{\prime} / t_{1}=0.4$, where there are wide and narrow bands, the interchain hopping dependence of several physical properties is similar to those for $t^{\prime} / t_{1}=0$ as shown in Fig. 2(b). For a larger interaction value of $U / t_{1}=8$, the variation of $n(0, \pi)$ against $t_{\mathrm{r}} / t_{1}$ becomes broad due to correlation effects as shown in Figs. 2(c) and 2(d). On the other hand, we find a clear 

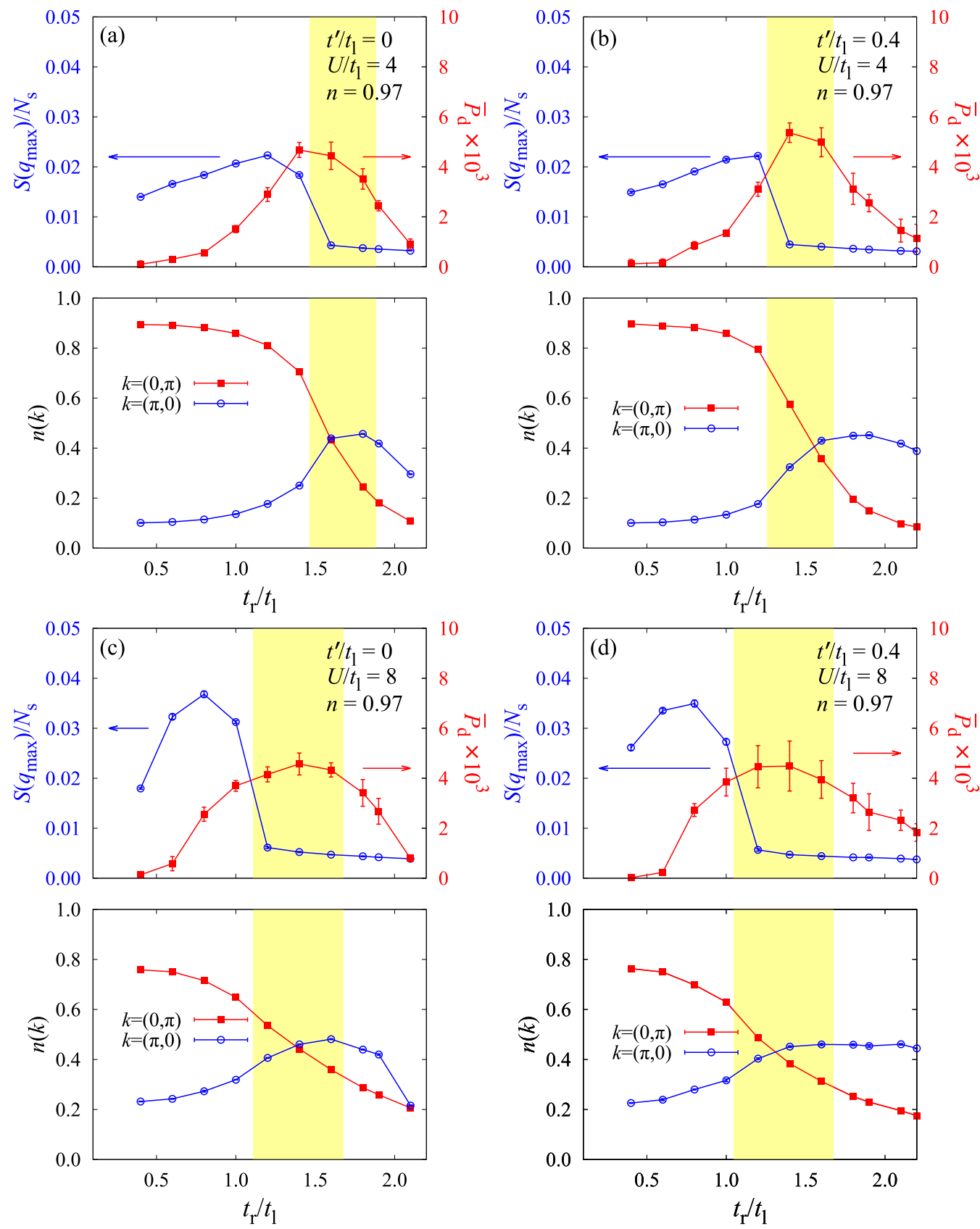

FIG. 2. Interchain hopping $t_{\mathrm{r}} / t_{1}$ dependence of the averaged partial $d$-wave superconducting correlation $\bar{P}_{d}$ and the peak value of the spin-structure factor $S\left(\boldsymbol{q}_{\max }\right)$ (upper panels), the momentum distribution function of the antibonding band minimum $n(0, \pi)$ and the bonding band maximum $n(\pi, 0)$ (lower panels) for the two-leg ladder model with (a) $t^{\prime} / t_{1}=0$ and $U / t_{1}=4$, (b) $t^{\prime} / t_{1}=0.4$ and $U / t_{1}=4$, (c) $t^{\prime} / t_{1}=0$ and $U / t_{1}=8$, and (d) $t^{\prime} / t_{1}=0.4$ and $U / t_{1}=8$. The band filling is $n=0.97$. The yellow region denotes the incipient band regime. In the present plots and the plots in the later figures, the error bars indicate the estimated statistical errors of the Monte Carlo sampling.

suppression of $S\left(\boldsymbol{q}_{\max }\right)$, which indicates the Lifshitz transition. Thus, superconductivity is optimized when a band becomes incipient also in the strongly correlated regime.

We also study $t_{\mathrm{r}} / t_{1}$ dependence of the superconducting correlation $\bar{P}_{d}$ for various values of $U / t_{1}$ as shown in Fig. 3. For both $t^{\prime} / t_{1}=0$ and $t^{\prime} / t_{1}=0.4$, the regime of enhanced superconducting correlation extends to smaller $t_{\mathrm{r}} / t_{1}$ as $U / t_{1}$ increases, presumably due to band narrowing caused by $U$. In general, the Hubbard $U$ can narrow bands near the Fermi level and induce the Lifshitz transition [53].

\section{B. Bilayer Hubbard model}

We next move on to the bilayer model. Figure 4(a) shows the interlayer hopping dependence of physical properties for $t_{\perp}^{\prime} / t=0$ and $U / t=8$, the peak value of the spin-structure factor $S\left(\boldsymbol{q}_{\max }\right)$, and the average value of the superconducting correlation $\bar{P}_{s^{ \pm}}$at long distances with the $s^{ \pm}$symmetry. We also plot the momentum distribution function at the antibonding band minimum $n(0,0, \pi)$ and the bonding band maximum $n(\pi, \pi, 0)$. For $t_{\perp} / t>1.8, n(0,0, \pi)$ decreases steeply, indicating the Fermi surface of the antibonding band 

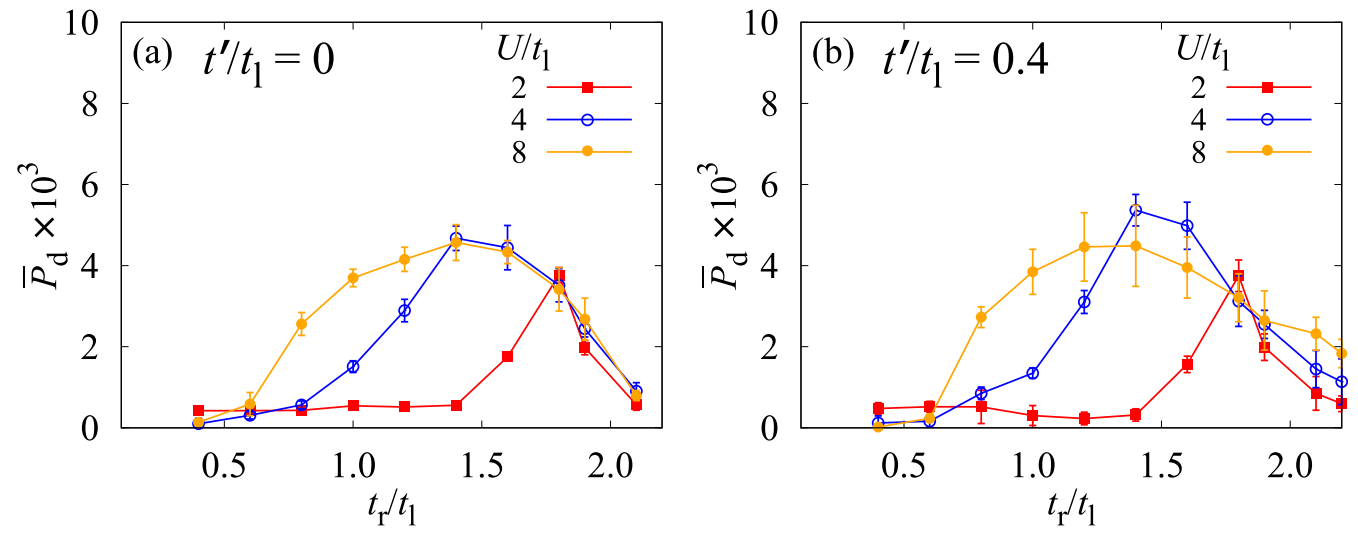

FIG. 3. $t_{\mathrm{r}} / t_{1}$ dependence of $\bar{P}_{d}$ for the two-leg ladder model with various values of $U / t_{1}$ and (a) $t^{\prime} / t_{1}=0$, (b) $t^{\prime} / t_{1}=0.4$. The band filling is $n=0.97$.

is lost, while $n(\pi, \pi, 0)$ barely varies and remains small, so that the Fermi level is well within the bonding band. Note that here an increase of $n(\pi, \pi, 0)$ is not observed, in contrast to the case of the $n(\pi, 0)$ in the two-leg ladder, presumably because the band filling is $n=0.94$, more away from half filling. Here again, $S\left(\boldsymbol{q}_{\max }\right)$ rapidly decreases for $t_{\perp} / t>1.8$. Thus, the incipient band regime is estimated to be in the range $1.8 \leqslant t_{\perp} / t \leqslant 2.4$. Around the incipient band regime, $\bar{P}_{s^{ \pm}}$is enhanced. The $s^{ \pm}$-wave superconducting correlation exhibits a dome structure around $t_{\perp} / t \sim 2.0$ [54], which is reminiscent of FLEX [31], functional renormalization group [35], and dynamical cluster approximation [36] studies. For $t_{\perp}^{\prime} / t=0.6, t_{\perp} / t$ dependence of the physical prop- erties is basically similar to those for $t_{\perp}^{\prime} / t=0$ as shown in Fig. 4(b)

As in the two-leg ladder, we also study $t_{\perp} / t$ dependence of the superconducting correlation $\bar{P}_{s^{ \pm}}$for various values of $U / t$ as shown in Fig. 5. For both $t_{\perp}^{\prime} / t=0$ and $t_{\perp}^{\prime} / t=0.6$, the regime where the superconducting correlation develops extends to lower $t_{\perp} / t$ as $U / t$ increases in the same way as the two-leg Hubbard ladder.

We note that $n(0,0, \pi)$ of the $U=8 t$ bilayer Hubbard model varies more steeply than $n(0, \pi)$ of the $U=8 t_{1}$ two-leg Hubbard ladder, and actually resembles that of $U=4 t_{1}$ ladder. Since the broadness of the momentum distribution variation around the Lifshitz transition presumably originates from the
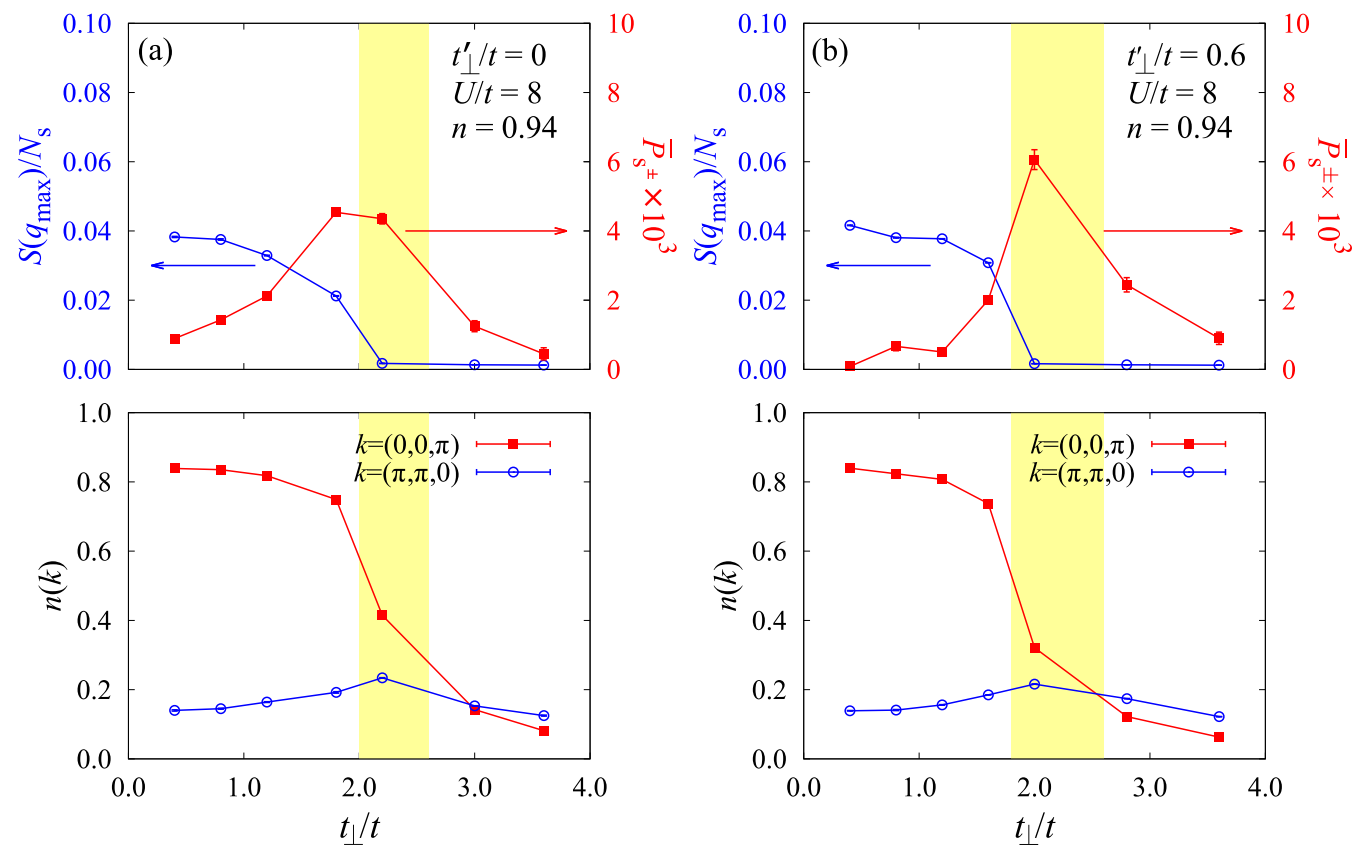

FIG. 4. Interlayer hopping $t_{\perp} / t$ dependence of the averaged $s^{ \pm}$-wave superconducting correlation $\bar{P}_{s^{ \pm}}$and the peak value of the spinstructure factor $S\left(\boldsymbol{q}_{\max }\right)$ (upper panels), and the momentum distribution functions of the antibonding minimum $n(0,0, \pi)$ and the bonding band maximum $n(\pi, \pi, 0)$ (lower panels) for the bilayer model with (a) $t_{\perp}^{\prime} / t=0$ and $U / t=8$ and (b) $t_{\perp}^{\prime} / t=0.6$ and $U / t=8$. The band filling is $n=0.94$. 

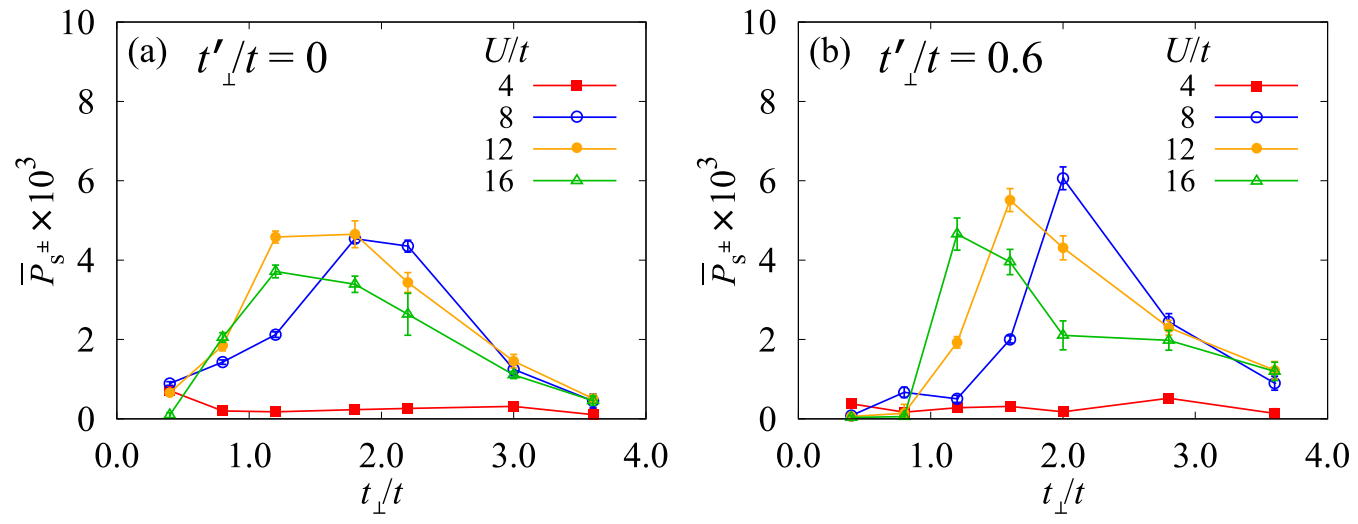

FIG. 5. $t_{\perp} / t$ dependence of $\bar{P}_{s^{ \pm}}$for the bilayer model with various values of $U / t$ and (a) $t_{\perp}^{\prime} / t=0$, (b) $t_{\perp}^{\prime} / t=0.6$. The band filling is $n=0.94$.

correlation effect, the present result indicates the strength of the electron correlation is roughly determined by $U / W$, where $W$ is the bandwidth.

\section{DISCUSSION}

The present results show that superconductivity is enhanced in the incipient band regime regardless of whether the system is one or two dimensional, or whether one of the bare bands is narrow or not. This is in fact reminiscent of the recent FLEX studies $[44,47,55]$. In this section, we further discuss the relation between the bilayer and two-leg ladder models, based on observations made in previous studies. References [37,38,47] pointed out the important role of the finite-energy spin fluctuations played in the enhancement of superconductivity in the bilayer Hubbard model. In particular, quite recently, in Ref. [47], the role played by the spin fluctuations in different energy ranges in two-band systems has been discussed based on the theory of Ref. [56]. Namely, the contribution from pair-breaking (pairing-effective) spin fluctuations scales as $1 / \omega^{4}\left(1 / \omega^{2}\right)$. Therefore, there is a critical frequency $\omega_{\mathrm{c}}$, smaller than a pairing cutoff energy $\varepsilon_{\mathrm{c}}$, so that the low-energy spin fluctuations with $\omega<\omega_{\mathrm{c}}$ lead to strong renormalization and hence are "pair breaking," while the finite-energy spin fluctuations with $\omega_{\mathrm{c}}<\omega<\varepsilon_{\mathrm{c}}$ enhance $T_{\mathrm{c}}$. Thus, when the low-lying spin fluctuations are suppressed while the finite-energy spin fluctuations are enhanced, superconductivity can be enhanced. In multiband systems, as one of the bands moves away from the Fermi level, the spinfluctuation spectral weight is transferred to higher energies. When the spin-fluctuation spectral weight is away from the critical frequency of spin fluctuations, but is within the pairing cutoff energy $\left(\omega_{\mathrm{c}}<\omega<\varepsilon_{\mathrm{c}}\right)$, the pairing interaction can be strong without strongly renormalizing the quasiparticles. On the other hand, when the spin fluctuations are concentrated at very low or too-high energies, superconductivity is degraded. From this viewpoint, we further discuss the relation between the superconducting correlation obtained by $\mathrm{mVMC}$ and the shape of the DOS of the antibonding band.

One difference between the bilayer and the two-leg ladder observed in the present study is the $U$ dependence of superconductivity. Namely, in the two-leg ladder, the super- conducting correlation is enhanced even for $U / t_{1}=2$ when the antibonding band is incipient, while such an enhancement is not obtained for the bilayer model for $U / t=4$. Note that here we compare two cases where $U$ normalized by the bare bandwidth are the same. Actually, a similar result was obtained in the recent FLEX calculation [47]. There, it has been pointed out that in the bilayer model, the correlation effect reduces the width of the incipient band [53], which makes more spin fluctuation weight lie within the energy regime effective for pairing. In the two-leg ladder, such an effect is not necessary when the antibonding band is incipient because in a one-dimensional system, the DOS is diverging at the band edge (see Fig. 6).

Another point that we notice, if we look closely, is that in the two-leg ladder, the superconducting correlation, maximized around the incipient band regime, is reduced as $t_{\mathrm{r}} / t_{1}$ decreases and the antibonding band intersects the Fermi level, but does so rather mildly and smoothly especially for $U=8 t_{1}$, whereas the reduction of the superconducting correlation in the bilayer model upon reducing $t_{\perp}$ occurs rapidly after the

\section{Two-leg ladder}
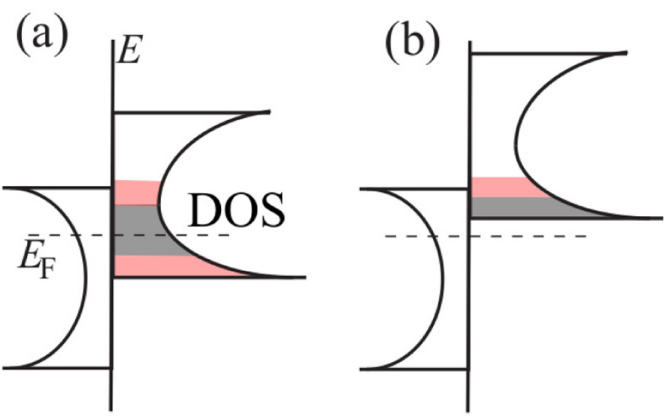

FIG. 6. Typical density of states of the two-leg ladder Hubbard model. In each part, the left (right) side of the vertical line depicts the DOS of the bonding (antibonding) band. The gray area denotes the portion of the bonding band DOS which gives rise to the low-lying pair-breaking spin fluctuations, and the red area is the portion of the antibonding band DOS contributing to the spin fluctuations which mediate pairings. (a) Both bands intersect the Fermi level and (b) the antibonding band is incipient. 


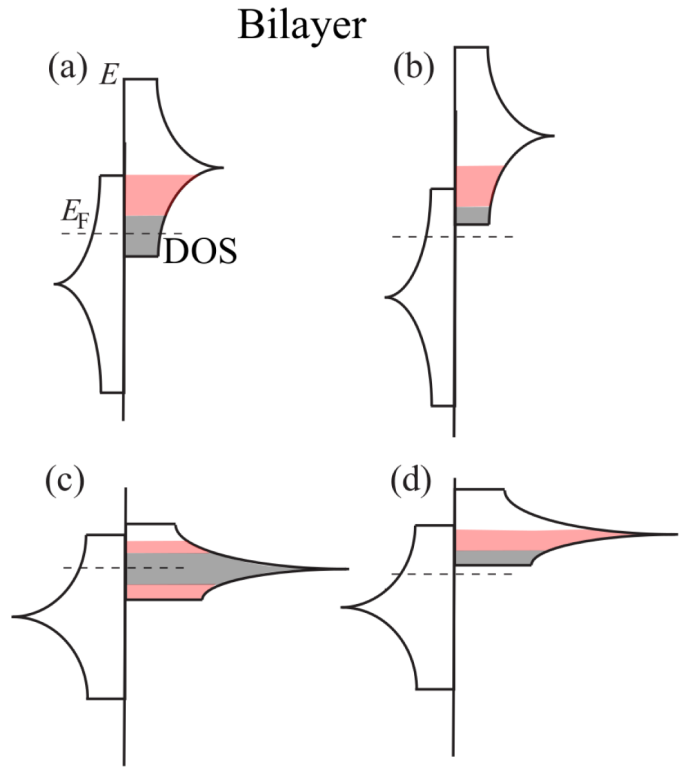

FIG. 7. Typical density of states for the bilayer Hubbard model: (a), (b) $t_{\perp}^{\prime} / t$ is (nearly) zero; (c), (d) $t_{\perp}^{\prime} / t$ is finite; (a), (c) both bands intersect the Fermi level; and (b), (d) the antibonding band is incipient.

antibonding band intersects the Fermi level. If we compare in more detail the two cases for the bilayer model, the reduction of the superconducting correlation is more abrupt for the case with $t_{\perp}^{\prime}=0.6 t$. These differences can again be understood from the shape of the DOS (see Figs. 6 and 7). Namely, in the two-leg ladder, where the DOS at the band edge is diverging, the DOS at the Fermi level decreases (rapidly, especially for large $U$ because the bandwidth shrinks due to renormalization) as $t_{\mathrm{r}}$ is reduced after the antibonding band intersects the Fermi level, so that the pair-breaking low-energy spin fluctuations become weaker. By contrast, in the bilayer model, where the DOS is diverging around the middle of the antibonding band, the DOS at the Fermi level increases upon reducing $t_{\perp}$ after the antibonding Fermi surface is formed, resulting in an increase of the pair-breaking spin fluctuations. The diverging DOS of the antibonding band approaches the Fermi level "faster" when $t_{\perp}^{\prime}$ is finite, so that the superconducting correlation is rapidly suppressed for the case of $t_{\perp}^{\prime}=0.6 t$ as $t_{\perp}$ is reduced. A similar analysis has been performed in Ref. [47], not for the $t_{\mathrm{r}}, t_{\perp}$ variation but for the $t^{\prime}\left(t_{\perp}^{\prime}\right)$ variation of superconductivity.

As mentioned above, the parameter dependence of the superconducting correlation function is reminiscent of the FLEX results obtained in Ref. [47]. However, this resemblance is highly nontrivial because it is not clear whether
FLEX, a weak-coupling approach, is valid for $U / t=6$ (a $U$ value comparable to the bandwidth) for which the study in Ref. [47] was performed. More importantly, the present VMC study has been performed for $U$ values as large as $U / t=$ 16. Therefore, the present study reveals that the following view holds, not only in the weak-coupling regime, but all the way up to the strong-coupling regime: superconductivity is enhanced by an incipient band due to the suppression of the near-zero-energy spin fluctuations and enhanced finite-energy spin fluctuations working as an effective pairing glue. It is important to note that, unlike FLEX, this conclusion is derived without an explicit consideration of the spin fluctuation as a pairing glue.

\section{SUMMARY}

To summarize, we have studied superconductivity in the Hubbard model on the two-leg ladder and bilayer square lattices. In both systems, superconductivity can be optimized in a region around the Lifshitz point, where one of the bands is (nearly) incipient [57]. The parameter dependence of the superconducting correlation function is reminiscent of the FLEX results obtained in Ref. [47]. Our study reveals that the following view holds even in the strong-coupling regime: superconductivity is enhanced by an incipient band owing to different roles played by spin fluctuations in different energy regimes, namely, the suppression of the near-zero-energy spin fluctuations and enhanced finite-energy spin fluctuations working as an effective pairing glue. We stress that the resemblance between the two approaches is highly nontrivial because the two approaches are totally different; FLEX is based on a weak-coupling perturbational theory, which takes into account the spin fluctuations (in momentum space) explicitly in the effective interaction, whereas the present $\mathrm{mVMC}$ method takes into account the electron correlation effect in a real-space-based manner, which is expected to be more appropriate in the strong-coupling regime. Since it has been shown that incipient bands enhance superconductivity in other models [41-46,53], it is an interesting future problem to study those models using the mVMC method.

\section{ACKNOWLEDGMENTS}

We appreciate Takahiro Misawa and Kota Ido for providing us guidance in the mVMC method. We also thank Masayuki Ochi, Karin Matsumoto, and Daisuke Ogura for fruitful discussions. The numerical calculations were performed at the following institutions: the Supercomputer Center, Institute for Solid State Physics, University of Tokyo, Yukawa Institute Computer Facility, Kyoto University, and the Cybermedia Center, Osaka University, provided through the HPCI System Research Project (Project ID hp190022).
[1] P. J. Hirschfeld, M. M. Korshunov, and I. I. Mazin, Gap symmetry and structure of Fe-based superconductors, Rep. Prog. Phys. 74, 124508 (2011).

[2] K. Kuroki, in Iron-Based Superconductors: Materials, Properties and Mechanisms (Pan Stanford Publishing, Singapore, 2013), Chap. 8.
[3] A. Chubukov and P. J. Hirschfeld, Iron-based superconductors, seven years later, Phys. Today 68(6), 46 (2015).

[4] H. Hosono and K. Kuroki, Iron-based superconductors: Current status of materials and pairing mechanism, Physica C 514, 399 (2015). 
[5] I. I. Mazin, D. J. Singh, M. D. Johannes, and M. H. Du, Unconventional Superconductivity with a Sign Reversal in the Order Parameter of $\mathrm{LaFeAsO}_{1-x} \mathrm{~F}_{x}$, Phys. Rev. Lett. 101, 057003 (2008).

[6] K. Kuroki, S. Onari, R. Arita, H. Usui, Y. Tanaka, H. Kontani, and H. Aoki, Unconventional Pairing Originating from the Disconnected Fermi Surfaces of Superconducting $\mathrm{LaFeAsO}_{1-x} \mathrm{~F}_{x}$, Phys. Rev. Lett. 101, 087004 (2008).

[7] J. Guo, S. Jin, G. Wang, S. Wang, K. Zhu, T. Zhou, M. He, and $X$. Chen, Superconductivity in the iron selenide $\mathrm{K}_{x} \mathrm{Fe}_{2} \mathrm{Se}_{2}(0 \leqslant$ $x \leqslant 1.0$ ), Phys. Rev. B 82, 180520(R) (2010).

[8] T. Qian, X.-P. Wang, W.-C. Jin, P. Zhang, P. Richard, G. Xu, X. Dai, Z. Fang, J.-G. Guo, X.-L. Chen et al., Absence of a Holelike Fermi Surface for the Iron-Based $\mathrm{K}_{0.8} \mathrm{Fe}_{1.7} \mathrm{Se}_{2}$ Superconductor Revealed by Angle-Resolved Photoemission Spectroscopy, Phys. Rev. Lett. 106, 187001 (2011).

[9] Q.-Y. Wang, Z. Li, W.-H. Zhang, Z.-C. Zhang, J.-S. Zhang, W. Li, H. Ding, Y.-B. Ou, P. Deng, K. Chang et al., Interfaceinduced high-temperature superconductivity in single unit-cell FeSe films on $\mathrm{SrTiO}_{3}$, Chin. Phys. Lett. 29, 037402 (2012).

[10] S. Tan, Y. Zhang, M. Xia, Z. Ye, F. Chen, X. Xie, R. Peng, D. $\mathrm{Xu}, \mathrm{Q}$. Fan, H. Xu et al., Interface-induced superconductivity and strain-dependent spin density waves in $\mathrm{FeSe} / \mathrm{SrTiO}{ }_{3}$ thin films, Nat. Mater. 12, 634 (2013).

[11] D. Liu, W. Zhang, D. Mou, J. He, Y.-B. Ou, Q.-Y. Wang, Z. Li, L. Wang, L. Zhao, S. He et al., Electronic origin of high-temperature superconductivity in single-layer FeSe superconductor, Nat. Commun. 3, 931 (2012).

[12] S. He, J. He, W. Zhang, L. Zhao, D. Liu, X. Liu, D. Mou, Y.-B. Ou, Q.-Y. Wang, Z. Li et al., Phase diagram and electronic indication of high-temperature superconductivity at $65 \mathrm{~K}$ in single-layer FeSe films, Nat. Mater. 12, 605 (2013).

[13] J. J. Lee, F. T. Schmitt, R. G. Moore, S. Johnston, Y.-T. Cui, W. Li, M. Yi, Z. K. Liu, M. Hashimoto, Y. Zhang et al., Interfacial mode coupling as the origin of the enhancement of $T_{\mathrm{c}}$ in FeSe films on $\mathrm{SrTiO}_{3}$, Nature (London) 515, 245 (2014).

[14] S. Iimura, S. Matsuishi, H. Sato, T. Hanna, Y. Muraba, S. W. Kim, J. E. Kim, M. Takata, and H. Hosono, Two-dome structure in electron-doped iron arsenide superconductors, Nat. Commun. 3, 943 (2012).

[15] H. Miao, T. Qian, X. Shi, P. Richard, T. Kim, M. Hoesch, L. Xing, X.-C. Wang, C.-Q. Jin, J.-P. Hu et al., Observation of strong electron pairing on bands without Fermi surfaces in $\mathrm{LiFe}_{1-x} \mathrm{Co}_{x} \mathrm{As}$, Nat. Commun. 6, 6056 (2015).

[16] X. H. Niu, R. Peng, H. C. Xu, Y. J. Yan, J. Jiang, D. F. $\mathrm{Xu}$, T. L. Yu, Q. Song, Z. C. Huang, Y. X. Wang et al., Surface electronic structure and isotropic superconducting gap in $\left(\mathrm{Li}_{0.8} \mathrm{Fe}_{0.2}\right) \mathrm{OHFeSe}$, Phys. Rev. B 92, 060504(R) (2015).

[17] A. Charnukha, D. V. Evtushinsky, C. E. Matt, N. Xu, M. Shi, B. Büchner, N. D. Zhigadlo, B. Batlogg, and S. V. Borisenko, High-temperature superconductivity from fine-tuning of Fermisurface singularities in iron oxypnictides, Sci. Rep. 5, 18273 (2015).

[18] Y. Miyata, K. Nakayama, K. Sugawara, T. Sato, and T. Takahashi, High-temperature superconductivity in potassiumcoated multilayer FeSe thin films, Nat. Mater. 14, 775 (2015).

[19] A. Kordyuk, Electronic band structure of optimal superconductors: From cuprates to ferropnictides and back again, Low Temp. Phys. 44, 477 (2018).
[20] F. Wang, F. Yang, M. Gao, Z.-Y. Lu, T. Xiang, and D.-H. Lee, The electron pairing of $\mathrm{K}_{x} \mathrm{Fe}_{2-y} \mathrm{Se}_{2}$, Europhys. Lett. 93, 57003 (2011).

[21] Y. Bang, A shadow gap in the over-doped $\left(\mathrm{Ba}_{1-x} \mathrm{~K}_{x}\right) \mathrm{Fe}_{2} \mathrm{As}_{2}$ compound, New J. Phys. 16, 023029 (2014).

[22] X. Chen, S. Maiti, A. Linscheid, and P. J. Hirschfeld, Electron pairing in the presence of incipient bands in iron-based superconductors, Phys. Rev. B 92, 224514 (2015).

[23] A. Linscheid, S. Maiti, Y. Wang, S. Johnston, and P. J. Hirschfeld, High $T_{\mathrm{c}}$ via Spin Fluctuations from Incipient Bands: Application to Monolayers and Intercalates of FeSe, Phys. Rev. Lett. 117, 077003 (2016).

[24] Y. Bang, Pairing mechanism of heavily electron doped FeSe systems: Dynamical tuning of the pairing cutoff energy, New J. Phys. 18, 113054 (2016).

[25] Y. Bang, Phonon boost effect on the $s^{ \pm}$-wave superconductor with incipient band, Sci. Rep. 9, 3907 (2019).

[26] N. Bulut, D. J. Scalapino, and R. T. Scalettar, Nodeless $d$-wave pairing in a two-layer Hubbard model, Phys. Rev. B 45, 5577 (1992).

[27] R. T. Scalettar, J. W. Cannon, D. J. Scalapino, and R. L. Sugar, Magnetic and pairing correlations in coupled Hubbard planes, Phys. Rev. B 50, 13419 (1994).

[28] R. E. Hetzel, W. von der Linden, and W. Hanke, Pairing correlations in a two-layer Hubbard model, Phys. Rev. B 50, 4159 (1994).

[29] R. R. dos Santos, Magnetism and pairing in Hubbard bilayers, Phys. Rev. B 51, 15540 (1995).

[30] A. I. Liechtenstein, I. I. Mazin, and O. K. Andersen, $s$-Wave Superconductivity from an Antiferromagnetic Spin-Fluctuation Model for Bilayer Materials, Phys. Rev. Lett. 74, 2303 (1995).

[31] K. Kuroki, T. Kimura, and R. Arita, High-temperature superconductivity in dimer array systems, Phys. Rev. B 66, 184508 (2002).

[32] S. S. Kancharla and S. Okamoto, Band insulator to Mott insulator transition in a bilayer Hubbard model, Phys. Rev. B 75 , 193103 (2007).

[33] K. Bouadim, G. G. Batrouni, F. Hebert, and R. T. Scalettar, Band insulator to Mott insulator transition in a bilayer Hubbard model, Phys. Rev. B 77, 144527 (2008).

[34] N. Lanata, P. Barone, and M. Fabrizio, Superconductivity in the doped bilayer Hubbard model, Phys. Rev. B 80, 224524 (2009).

[35] H. Zhai, F. Wang, and D.-H. Lee, Antiferromagnetically driven electronic correlations in iron pnictides and cuprates, Phys. Rev. B 80, 064517 (2009).

[36] T. A. Maier and D. J. Scalapino, Pair structure and the pairing interaction in a bilayer Hubbard model, Phys. Rev. B 84, 180513(R) (2011).

[37] V. Mishra, D. J. Scalapino, and T. Maier, $s_{ \pm}$pairing near a Lifshitz transition, Sci. Rep. 6, 32078 (2016)

[38] M. Nakata, D. Ogura, H. Usui, and K. Kuroki, Finite-energy spin fluctuations as a pairing glue in systems with coexisting electron and hole bands, Phys. Rev. B 95, 214509 (2017).

[39] T. A. Maier, V. Mishra, G. Balduzzi, and D. J. Scalapino, Effective pairing interaction in a system with an incipient band, Phys. Rev. B 99, 140504(R) (2019).

[40] K. Kuroki, T. Higashida, and R. Arita, High- $T_{\mathrm{c}}$ superconductivity due to coexisting wide and narrow bands: A fluctuation exchange study of the Hubbard ladder as a test case, Phys. Rev. B 72, 212509 (2005). 
[41] K. Kobayashi, M. Okumura, S. Yamada, M. Machida, and H. Aoki, Superconductivity in repulsively interacting fermions on a diamond chain: Flat-band-induced pairing, Phys. Rev. B 94, 214501 (2016).

[42] T. Misumi and H. Aoki, New class of flat-band models on tetragonal and hexagonal lattices: Gapped versus crossing flat bands, Phys. Rev. B 96, 155137 (2017).

[43] D. Ogura, H. Aoki, and K. Kuroki, Possible high- $T_{\mathrm{c}}$ superconductivity due to incipient narrow bands originating from hidden ladders in Ruddlesden-Popper compounds, Phys. Rev. B 96, 184513 (2017).

[44] K. Matsumoto, D. Ogura, and K. Kuroki, Wide applicability of high- $T_{\mathrm{c}}$ pairing originating from coexisting wide and incipient narrow bands in quasi-one-dimensional systems, Phys. Rev. B 97, 014516 (2018).

[45] S. Sayyad, E. W. Huang, M. Kitatani, M.-S. Vaezi, Z. Nussinov, A. Vaezi, and H. Aoki, Pairing and non-Fermi liquid behavior in partially flat-band systems, Phys. Rev. B 101, 014501 (2020).

[46] H. Aoki, Theoretical possibilities for flat-band superconductivity, arXiv:1912.04469.

[47] K. Matsumoto, D. Ogura, and K. Kuroki, Strongly enhanced superconductivity due to finite energy spin fluctuations induced by an incipient band: A FLEX study on the bilayer Hubbard model with vertical and diagonal interlayer hoppings, J. Phys. Soc. Jpn. 89, 044709 (2020).

[48] D. Tahara and M. Imada, Variational Monte Carlo method combined with quantum-number projection and multi-variable optimization, J. Phys. Soc. Jpn. 77, 114701 (2008).

[49] T. Misawa, S. Motira, K. Yoshimi, M. Kawamura, Y. Motoyama, K. Ido, T. Ohgoe, M. Imada, and T. Kato, mVMC-open-source software for many-variable variational Monte Carlo method, Comput. Phys. Commun. 235, 447 (2019).

[50] Recently, M. Kainth and M. W. Long, arXiv:1904.07138, studied superconductivity in the bilayer Hubbard model with equal horizontal intralayer and diagonal interlayer hoppings (the case with a flat band) in the strong-coupling regime using a nonlinear fermion transformation.

[51] S. Sorella, Generalized Lanczos algorithm for variational quantum Monte Carlo, Phys. Rev. B 64, 024512 (2001).
[52] K. Kuroki and R. Arita, Possible high- $T_{\mathrm{c}}$ superconductivity mediated by antiferromagnetic spin fluctuations in systems with Fermi surface pockets, Phys. Rev. B 64, 024501 (2001).

[53] D. Ogura, Springer Theses: Theoretical Study of Electron Correlation Driven Superconductivity in Systems with Coexisting Wide and Narrow Bands (Springer, Berlin, 2019).

[54] The $d_{x^{2}-y^{2}}$ pairing dominates over the $s^{ \pm}$pairing only for small $t_{\perp}(\leqslant 0.8 t)$ if $d$-wave states are employed as initial states.

[55] Some notations used in the present study are different from those in Ref. [47]. $t^{\prime}$ and $t_{\perp}^{\prime}$ in the present study correspond to $-t^{\prime}$ in Ref. [47]. Therefore, in the present study, the antibonding band is the narrow band, whereas in Ref. [47], the bonding band is the narrow band. To make the narrow band incipient with band fillings close to half filling, in the present study, band fillings smaller than half-filling are taken to consider cases where the narrow antibonding band lies above the Fermi level in the incipient band situation. On the other hand, in Ref. [47], band fillings larger than half-filling were taken so as to make the narrow bonding band lie below the Fermi level. In short, the cases considered here and those in Ref. [47] are equivalent and connected by electron-hole transformation. Also, the definition of the band filling is different; $n$ in the present study corresponds to $n / 2$ in Ref. [47].

[56] A. J. Millis, S. Sachdev, and C. M. Varma, Inelastic scattering and pair breaking in anisotropic and isotropic superconductors, Phys. Rev. B 37, 4975 (1988).

[57] This might seem reminiscent of spin-fluctuation mediated superconductivity enhanced near a quantum critical point (QCP) of antiferromagnetic ordering, for which apparently similar numerical results are obtained as in, e.g., E. Berg, M. A. Metlitski, and S. Sachdev, Science 338, 1606 (2012). We note, however, that the incipient band situation is actually far from the QCP of antiferromagnetic ordering. Although this cannot be directly probed by the present $\mathrm{mVMC}$ calculation, it can be confirmed from the FLEX calculation results of the dynamical spin susceptibility in Ref. [47] (Fig. 6 of Ref. [47]), which show that the spin fluctuations in the very low energy regime are suppressed in the incipient band case. This is reinforced by the calculation of the Stoner factor of antiferromagnetism in Ref. [53], which is far from unity in the incipient band situation of the bilayer model (Fig. 4.7 of Ref. [53]). 